\title{
Grasp Analysis and Synthesis of 2D Articulated Objects with $n$ Links
}

\author{
Noé Alvarado Tovar, Raúl Suárez \\ Institut d'Organizació i Control de Sistemes Industrials (IOC) \\ Universitat Politècnica de Catalunya (UPC) \\ Barcelona, Spain
}

\begin{abstract}
This paper proposes a solution to the problem of grasp analysis and synthesis of 2D articulated objects with $n$ links considering frictionless contacts. The boundary of each link of the object is represented by a finite set of boundary points allowing links of any shape to be considered. Grasp analysis is carried out to verify whether a set of contact points on the object boundary allows a force-closure grasp, while the goal of grasp synthesis is to determine a set of contact points that allows a force-closure grasp. The paper describes the process of finding the elements of the generalized wrench vector generated by a force applied to any link of the articulated object and a procedure to search for a force-closure grasp based on these generalized wrenches. The approach has been implemented and some examples are included in the paper.
\end{abstract}

Keywords: Grasping, Force-Closure Grasps, Grasp Analysis, Grasp Synthesis

\section{Introduction}

The majority of robots used in industry, at home, at school, or in research carry out activities or operations which require grasping, fixing or manipulating objects of different shapes and sizes. Moreover, many of these objects may be articulated, i.e. composed of rigid links connected by joints or hinges, such as scissors, staplers, doors, laptop computers, pliers, truck toys and some cell phones. The goal of a grasp is to constrain the object degrees of freedom despite the possible presence of external force disturbances (see [1] for a survey on grasping).

Typically, a grasp must satisfy one of the following properties: a) force-closure (hereafter FC), which means that the forces applied by the fingers ensure object immobility; or b) form-closure, which means that the finger contact positions on the object boundary ensure object immobility [2]. In the case of a 2D rigid body with three degrees of freedom grasped by fingers pressing the object at punctual contacts, four fingers are necessary to ensure an FC grasp if the contacts are frictionless while only two fingers may be enough if the contacts are frictional. Regarding form-closure immobilization, since the fingers have fixed positions and do not push against the object, friction is not relevant and four punctual contacts are always necessary. It must however, be remarked that when the object has rotational symmetry, frictionless contacts do not allow its immobilization. A detailed discussion about the number of contact points necessary for each case can be found in [3] [4]. 
Several works deal with FC grasps of 2D objects, both polygonal objects with three frictional contacts [5][6] and four frictionless contacts [7][8], and non-polygonal objects with four frictionless contacts [9] and three frictional contacts [10]. The problem has also been adressed for 3D objects, both polyhedra with three [11] and four [12] frictional contacts and objects of any shape with seven [13] frictionless contacts and three [14][15] and four [16] frictional contacts.

Nevertheless, the majority of the work done in the area of object grasping and fixturing was centered on 2D or 3D solid object while grasp and manipulation of non-rigid objects were lees common. For instance, there are works dealing with the immobilization of deformable 2D objects using two frictionless [17] and two frictional [18] contacts. There are also works dealing with the immobilization of non-stretchable cloth polygons [19], showing that it is necessary to pin all the convex vertices plus no more than one-third of the concave vertices for simple polygons, or plus no more than onethird of the concave vertices and two additional pins per hole for polygons with holes. Dealing with grasping and fixturing of articulated objects, the topic o this paper, there are relevant works based on the use of spatial operator algebra algorithms for modeling and dynamic analysis of multiple parallel manipulators grasping an articulated object [20], interactive perception algorithms [21] or an occlusion aware reconstruction system [22] to acquire a model that enables the manipulation of articulated objects by a robot. Although these works proposed approaches to the grasping and manipulation of articulated objects, they did not present a systematic procedure to find a set of points on the object boundary that allow an FC grasp or a test to check whether a given grasp is FC.

Regarding immobilization by frictionless contacts of a 2D serial chain with $n$ polygons linked by joints with one degree of freedom, it was determined that the number of sufficient contacts is:

- Polygons without parallel edges [23]: $n+2$ contacts if $n \neq 3$, and $n+3$ contacts otherwise.

- Polygons with parallel edges [24]: $n+2$ contacts if $n$ is even, and $n+3$ if $n$ is odd.

In order to achieve robust immobilization (i.e. any contact can be perturbed slightly without loss of the immobilization) when these chains have $n \geq 6$ polygons, the following strategies were used [23] [24]:

- Polygons without parallel edges: the chain is divided into sets of five polygons starting from one end of the chain until at most five polygons are left. Then, each group of five polygons is immobilized with six contacts with the arrangement $(0,0,3,0,3)$, which means 0 contacts in the first, second and fourth polygons, and three contacts in the third and fifth polygons. The remaining polygons are immobilized with a number of contacts that depends on the number of remaining polygons.

- Polygons of any type (i.e. they may have parallel edges): the chain is divided into sets of four polygons until at most four polygons are left. Then, each group of four polygons is immobilized with five contacts using the arrangement $(0,0,3,2)$, 
which means zero contacts in the first and second polygons, three contacts in the third polygon and two contacts in fourth polygon. The remaining polygons (except if these are three of them) are immobilized as above described for chains of polygons without parallel edges. If three polygons are left, they are combined with the last quadruple and robustly immobilized with the arrangement $(4,0,0,4,0,0,4)$, i.e. four contacts in the first, fourth and seventh polygons, and 0 contacts in the second, third, fifth and sixth polygons.

More recently, based on second-order effects, the upper bound was demonstrated to be $n+2$ for any chain of $n \neq 3$ hinged polygons without parallel edges. In any case, $n+3$ frictionless contact points are always sufficient to immobilize any chain of $n$ polygons [25].

In this work, we focus on general FC grasps of 2D serial articulated objects with $n$ links and $m=n+2$ degrees of freedom (dof) considering the minimum number of frictionless contacts $k=m+1=n+3$. We first deal with the problem of determining whether a given set of contact points on an articulated object allows an FC grasp, and second with the problem of finding FC grasps. In latter, a random initial grasp is found, and if it is not FC, then the contact points are iteratively changed to search for an FC grasp.

The algorithms developed here are based on the work by Roa and Suárez [13], extending it to the case of articulated objects. Both in grasp analysis and grasp synthesis, the generalized force vector plays a relevant role.Thus, a procedure to find a proper representation of this vector is presented.

The contribution of this work is a systematic procedure to analyze and synthesize FC grasps of articulated objects using a generalized wrench space. The grasping device is not considered, unlike in [26][27][28] for rigid objects. Hence, in the grasp synthesis the proposed contact points may not be reachable by certain type of robotic hands, but they are always useful for object fixturing in industrial applications.

The rest of the paper is structured as follows. Section II provides an overview of the problem, including the main assumptions. Section III presents a procedure to find the vector elements of generalized wrenches for an articulated object with $n$ links. Section IV details an analysis to determine whether a given set of contacts points allows a FC grasp. Section V describes the algorithm to find an FC grasp. Section VI shows some examples of the proposed approach. Finally, Section VI presents some conclusions and proposes future work.

\section{Problem Definition and Assumptions}

Consider a 2D serial articulated object with $n$ links and rotational joints, as illustrated in Fig. 1. The problems to be addressed are as follows:

- Determination of whether a given set of contact points on the surface of the links allows an FC grasp of the object, i.e. they allow the immobilization the object, including the internal dof, in the presence of external perturbations.

- Search for a set of contact points on the surface of the links that allows a FC grasp. 


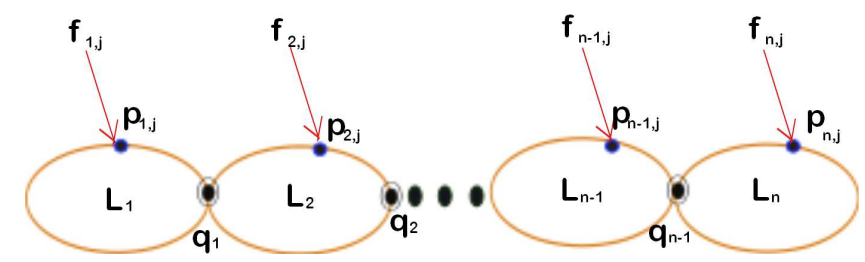

Figure 1: Articulated object with $n$ links (a generic force $\boldsymbol{f}_{i, j}$ acting on a point $\boldsymbol{p}_{i, j}$ is represented on each link $i$ ).

The following assumptions are considered:

- The links are connected by rotational joints.

- The links can overlap each other. This does not pose any problem.

- The boundary of each link is represented by a (large enough) set $\Omega$ of points described by position vectors $\boldsymbol{p}_{i, j}$ (therefore, the proposed approach is valid independently of whether the links are polygonal or non-polygonal).

- The normal direction $\hat{\boldsymbol{n}}_{i, j}$ pointing towards the interior of the object at each point $\boldsymbol{p}_{i, j}$ is known.

- The contact points between the fingers and the object are frictionless. This ensures a worst-case grasp, because the existence of friction in real cases will increase grasp robustness.

\section{Generalized wrenches for articulated objects}

\subsection{Generalized wrenches for a rigid body}

Consider a coordinate system at the center of mass (COM) of the object used to describe the positions $\boldsymbol{p}_{i}$ of the contact points. A force $\boldsymbol{f}_{i}$ applied to the object at $\boldsymbol{p}_{i}$ generates a torque $\boldsymbol{\tau}_{i}=\boldsymbol{p}_{i} \times \boldsymbol{f}_{i}$ with respect to COM; $\boldsymbol{f}_{i}$ and $\boldsymbol{\tau}_{i}$ can be grouped in a wrench vector $\boldsymbol{w}_{i}=\left(\boldsymbol{f}_{i}, \boldsymbol{\tau}_{i}\right)^{T}$. For frictionless grasps, forces can only be applied in the direction normal to the object boundary. Thus, the wrench vector is given by

$$
\boldsymbol{w}_{i}=\left[\begin{array}{c}
\boldsymbol{f}_{i} \\
\boldsymbol{\tau}_{i}
\end{array}\right]=\left[\begin{array}{c}
\boldsymbol{f}_{i} \\
\boldsymbol{p}_{i} \times \boldsymbol{f}_{i}
\end{array}\right]=f_{i}\left[\begin{array}{c}
\hat{\boldsymbol{n}}_{i} \\
\boldsymbol{p}_{i} \times \hat{\boldsymbol{n}}_{i}
\end{array}\right]
$$

with $f_{i}$ being the magnitude of $\boldsymbol{f}_{i}$.

A grasp defined by a set of $k$ frictionless contacts, $G=\left\{\boldsymbol{p}_{1}, \ldots, \boldsymbol{p}_{k}\right\}$, is able to apply $k$ wrenches $\boldsymbol{w}_{i}$ to the object that can be grouped in a wrench set $W=\left\{\boldsymbol{w}_{1}, \ldots, \boldsymbol{w}_{k}\right\}$. The information in $W$ is sufficient to analyze whether $G$ allows a FC grasp, which can 
now be formally defined as a grasp able to apply forces $\boldsymbol{f}_{i}$ producing wrenches $\boldsymbol{w}_{i}$ that counterbalance any perturbation $\boldsymbol{w}_{e}$ in the object, i.e.

$$
\boldsymbol{w}_{e}=\sum_{i=1}^{k} \boldsymbol{w}_{i}, \forall \boldsymbol{w}_{e}
$$

Then, for planar objects with three dof the wrench vectors $\boldsymbol{w}_{i}$ are 3-dimensional, and so is the wrench space. In the absence of rotational symmetries, four contacts are sufficient to ensure the FC condition, i.e. a set of points $G=\left\{\boldsymbol{p}_{1}, \ldots, \boldsymbol{p}_{4}\right\}$ allowing an appropriate set of wrenches $W=\left\{\boldsymbol{w}_{1}, \ldots, \boldsymbol{w}_{4}\right\}$ always allows an FC grasp [3] [4].

\subsection{Generalized wrenches for a serial articulated object}

The generalized wrench vector generated by the application of a force to a link of the articulated object is deduced by a general analysis of an open virtual kinematic chain with $n+2$ links. Fig. 2 illustrates a virtual robot with $n+2$ links that contains the articulated object and some auxiliary elements (two virtual links and three virtual joints) used for the developments below. Virtual kinematic chains were also used in [29] to generate a systematic constraint-based approach to specify complex tasks of general sensor-based robot systems consisting of rigid links.

The following basic nomenclature is used in the rest of the paper(see Fig. 2):

$L_{i}$ : Link $i$ of the virtual robot, $i=-1, \ldots, n$. Note that links -1 and 0 are virtual ones, and links 1 to $n$ correspond to the real articulated object.

$q_{i}$ : Joint $i$ of the virtual robot, $i=-2, \ldots, n-1$ (generalized coordinates). Note that joints $-2,-1$ and 0 are virtual ones, and joints 1 to $n-1$ correspond to the joints of real articulated object.

$Q_{i}$ : Origin of reference frame attached to link $i, i=-1, \ldots, n$. These are equivalent to the positions of the joints $q_{i}, i=-1, \ldots, n-1$, and position of the final end of the link $L_{n}$ for $i=n$, respect to the base frame.

$\boldsymbol{P}_{i, j}$ : Contact point $j$ on link $L_{i}$ respect to the base frame.

$\boldsymbol{p}_{i, j}$ : Contact point $j$ on link $L_{i}$ represented with respect to joint $q_{i-1}$ (i.e. $\boldsymbol{p}_{i, j}=\boldsymbol{P}_{i, j}-\boldsymbol{Q}_{i}$ ), $i=-1, \ldots, n, j=1, \ldots, k_{i}$, where $k_{i}$ is the number of contact points on link $L_{i}$. Note that the total number of contacts is $k=\sum_{i} k_{i}$.

$\boldsymbol{r}_{i}$ : Position $\boldsymbol{Q}_{i}$ respect $\boldsymbol{Q}_{i-1}$ (i.e. $\boldsymbol{r}_{i}=\boldsymbol{Q}_{i}-\boldsymbol{Q}_{i-1}$ ).

$\boldsymbol{s}_{i, j}$ : Contact point $j$ on link $L_{i}$ respect to $\boldsymbol{Q}_{i}$ (i.e. $\boldsymbol{s}_{i, j}=\boldsymbol{P}_{i, j}-\boldsymbol{Q}_{i}=\boldsymbol{p}_{i, j}-\boldsymbol{r}_{i}$ ).

$\boldsymbol{f}_{i, j}:$ Force $j$ applied to link $L_{i}$ at contact point $\boldsymbol{p}_{i, j}$.

$\boldsymbol{W}_{i, j}$ : generalized wrench produced by force $\boldsymbol{f}_{i, j}$ applied to $\boldsymbol{p}_{i, j}$. 


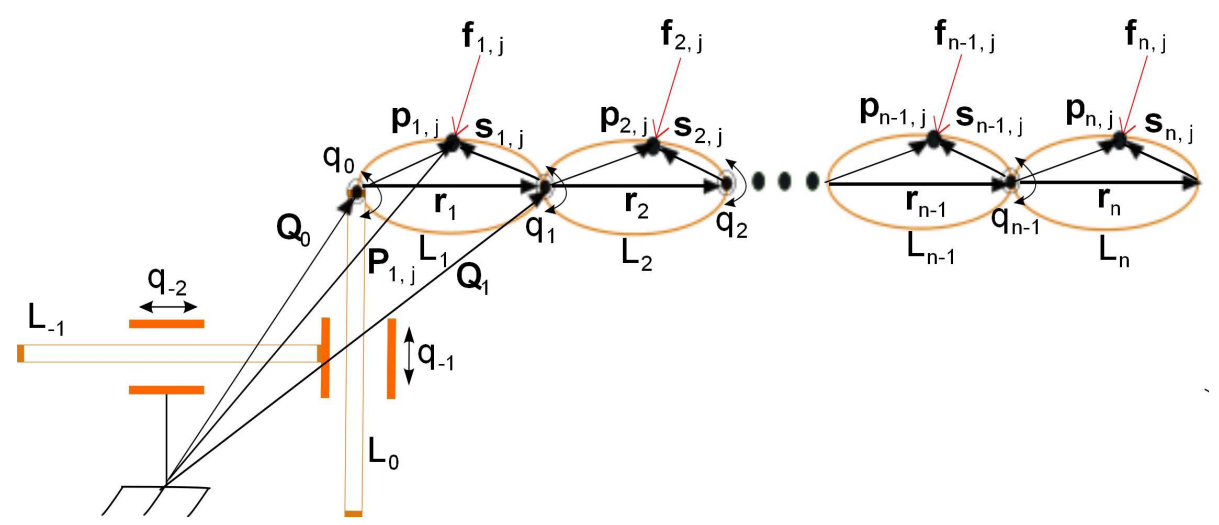

Figure 2: Schematic diagram of the virtual robot, where links $L_{-1} \ldots L_{n}$ represent the total links of the virtual robot and $L_{1} \ldots L_{n}$ are the links of the articulated object.

The generalized wrench vector is obtained as follows. Consider the virtual robot with $n+2$ links in Fig. 2, where the first two links $\left(L_{-1}\right.$ and $\left.L_{0}\right)$ are virtual ones and the rest are equivalent to the articulated object to be grasped. The first three joints $\left(q_{-2}, q_{-1}\right.$ and $\left.q_{0}\right)$ are therefore not real, but they are useful for the model development because they represent the three degrees of freedom of the first link of the chain, while each real joint represents one of the internal degrees of freedom of the object. $q_{-2}$ and $q_{-1}$ are prismatic joints and $q_{0}$ is revolute; the remaining joints $q_{i}, i=1, \ldots n-1$, are those of the articulated object.

Fig. 2 shows a general force $\boldsymbol{f}_{i, j}$ applied to each link $L_{i}$ of the articulated object at a point $\boldsymbol{p}_{i, j}$, which can be expressed as

$$
\boldsymbol{f}_{i, j}=\left[\begin{array}{l}
f_{x_{i, j}} \\
f_{y_{i, j}}
\end{array}\right]
$$

$\boldsymbol{f}_{i, j}$ and the resultant moment with respect to $Q_{i}$ are grouped into a wrench vector $\boldsymbol{w}_{i, j}$ acting on $L_{i}$ as

$$
\boldsymbol{w}_{i, j}=\left[\begin{array}{c}
f_{x_{i, j}} \\
f_{y_{i, j}} \\
M_{s_{i, j}}
\end{array}\right]
$$

where $M_{s_{i, j}}=s_{i, j} \times \boldsymbol{f}_{i, j}$.

Consider the Jacobian $J_{i}$ for each link $L_{i}$ of the virtual robot in order to relate forces $\boldsymbol{f}_{i, j}$ applied to link $L_{i}$ with torques $\tau_{k}$ at each robot joint under equilibrium condition (Jacobian $J_{i}$ is computed by standard procedures used for serial robots [30][31]).

Then, the vector $\boldsymbol{\tau}_{i, j}$ of torques $\boldsymbol{\tau}_{k_{i, j}}$ at joints $q_{k}$ necessary to balance the effect of a wrench $\boldsymbol{w}_{i, j}$ produced by a force $\boldsymbol{f}_{i, j}$ applied on the link $L_{i}$ is obtained as

$$
\boldsymbol{\tau}_{i, j}=\left[\tau_{-2_{i, j}}, \ldots, \tau_{k_{i, j}}, \ldots, \tau_{(n-1)_{i, j}}\right]^{T}=J_{i}^{T} \boldsymbol{w}_{i, j}=J_{i}^{T}\left[\begin{array}{c}
f_{x_{i, j}} \\
f_{y_{i, j}} \\
M_{s_{i, j}}
\end{array}\right]
$$


Then, the vector $\tau_{i}$ of torques $\tau_{k_{i}}$ in joints $q_{k}$ due to all the forces $j$ applied to $L_{i}$ results in

$$
\boldsymbol{\tau}_{i}=\left[\tau_{-2_{i}}, \ldots, \tau_{k_{i}}, \ldots, \tau_{(n-1)_{i}}\right]^{T}=\sum_{j=1}^{k_{i}} \boldsymbol{\tau}_{i, j}=\sum_{j=1}^{k_{i}} J_{i}^{T} \boldsymbol{w}_{i, j}=\sum_{j=1}^{k_{i}} J_{i}^{T}\left[\begin{array}{l}
f_{x_{i, j}} \\
f_{y_{i, j}} \\
M_{s_{i, j}}
\end{array}\right]
$$

and the torques $\tau_{k}$ in joints $q_{k}$ due all forces $\boldsymbol{f}_{i, j}$ applied to all the links $L_{i}$ is given by

$\boldsymbol{\tau}=\left[\tau_{-2}, \ldots, \tau_{k}, \ldots, \tau_{(n-1)}\right]^{T}=\sum_{i=-1}^{n} \sum_{j=1}^{k_{i}} \boldsymbol{\tau}_{i, j}=\sum_{i=-1}^{n} \sum_{j=1}^{k_{i}} J_{i}^{T} \boldsymbol{w}_{i, j}=\sum_{i=-1}^{n} \sum_{j=1}^{k_{i}} J_{i}^{T}\left[\begin{array}{l}f_{x_{i, j}} \\ f_{y_{i, j}} \\ M_{s_{i, j}}\end{array}\right]$

Forces $\boldsymbol{f}_{i, j}$ acting on the virtual robot include external perturbation forces and those applied by the grasping device (it must be noted that, since $L_{-1}$ and $L_{0}$ are virtual links, applying forces to them is not possible, i.e. $\boldsymbol{f}_{-1, j}=\boldsymbol{f}_{0, j}=0$ ). Since it is desired to immobilize the articulated object, the total torque at each joint must be null (considering perturbations and forces applied by the fingers), i.e. $\boldsymbol{\tau}=\mathbf{0}$, and therefore $\tau_{k}=0 \forall k$.

Then, computing Jacobians $J_{i}$ and making $\tau_{k}=0, \forall k$, from eq. (7) we obtain:

$$
\begin{aligned}
& \tau_{-2}=\sum_{j} f_{x_{1, j}}+\sum_{j} f_{x_{2, j}}+\ldots+\sum_{j} f_{x_{n-1, j}}+\sum_{j} f_{x_{n, j}}=0 \\
& \tau_{-1}=\sum_{j} f_{y_{1, j}}+\sum_{j} f_{y_{2, j}}+\ldots+\sum_{j} f_{y_{n-1, j}}+\sum_{j} f_{y_{n, j}}=0 \\
& \tau_{0}=\sum_{j} \boldsymbol{p}_{1, j} \times \boldsymbol{f}_{1, j}+\sum_{j} \boldsymbol{r}_{1} \times \boldsymbol{f}_{2, j}+\ldots+\sum_{j} \boldsymbol{r}_{1} \times \boldsymbol{f}_{n-1, j}+\sum_{j} \boldsymbol{r}_{1} \times \boldsymbol{f}_{n, j}=0 \\
& \tau_{1}=\begin{array}{cc}
0 & \sum_{j} \boldsymbol{p}_{2, j} \times \boldsymbol{f}_{2, j}+\ldots+\sum_{j} \boldsymbol{r}_{2} \times \boldsymbol{f}_{n-1, j}+\sum_{j} \boldsymbol{r}_{2} \times \boldsymbol{f}_{n, j}=0
\end{array} \\
& \vdots \quad \vdots \quad 0 \\
& \begin{array}{ccccc}
\tau_{n-2}= & 0 & \vdots & \ldots & \sum_{j} \boldsymbol{p}_{n-1, j} \times \boldsymbol{f}_{n-1, j}+\sum_{j} \boldsymbol{r}_{n-1} \times \boldsymbol{f}_{n, j}=0 \\
\tau_{n-1}= & 0 & 0 & \ldots & 0 \\
\sum_{j} \boldsymbol{p}_{n, j} \times \boldsymbol{f}_{n, j} & =0
\end{array}
\end{aligned}
$$

Now, it is possible to consider a generalized wrench space $\mathcal{W}$ defined by the base $\left\{\tau_{-2}, \tau_{-1}, \tau_{0}, \tau_{1}, \ldots, \tau_{2}, \tau_{n-1}\right\}$, such that the generalized wrenches $\boldsymbol{W}_{1, j}, \boldsymbol{W}_{2, j}, \ldots$, $\boldsymbol{W}_{n-1, j}, \boldsymbol{W}_{n, j}$ generated, respectively, by forces $\boldsymbol{f}_{1, j}, \boldsymbol{f}_{2, j}, \ldots, \boldsymbol{f}_{n-1, j}, \boldsymbol{f}_{n, j}$ are

$$
\begin{gathered}
\boldsymbol{W}_{1, j}=\left[\begin{array}{c}
f_{x_{1, j}} \\
f_{y_{1, j}} \\
\boldsymbol{p}_{1, j} \times \boldsymbol{f}_{1, j} \\
0 \\
\vdots \\
0 \\
0
\end{array}\right] \boldsymbol{W}_{2, j}=\left[\begin{array}{c}
f_{x_{2, j}} \\
f_{y_{2, j}} \\
\boldsymbol{r}_{1} \times \boldsymbol{f}_{2, j} \\
\boldsymbol{p}_{2, j} \times \boldsymbol{f}_{2, j} \\
0 \\
\vdots \\
0 \\
0
\end{array}\right] \\
\boldsymbol{W}_{n-1, j}=\left[\begin{array}{c}
f_{x_{n-1, j}} \\
f_{y_{n-1, j}} \\
\boldsymbol{r}_{1} \times \boldsymbol{f}_{n-1, j} \\
\boldsymbol{r}_{2} \times \boldsymbol{f}_{n-1, j} \\
\vdots \\
\boldsymbol{r}_{n-2} \times \boldsymbol{f}_{n-1, j} \\
\boldsymbol{p}_{n-1, j} \times \boldsymbol{f}_{n-1, j} \\
0
\end{array}\right] \boldsymbol{W}_{n, j}=\left[\begin{array}{c}
f_{x_{n, j}} \\
f_{y_{n, j}} \\
\boldsymbol{r}_{1} \times \boldsymbol{f}_{n, j} \\
\boldsymbol{r}_{2} \times \boldsymbol{f}_{n, j} \\
\vdots \\
\vdots \\
\boldsymbol{r}_{n-1} \times \boldsymbol{f}_{n, j} \\
\boldsymbol{p}_{n, j} \times \boldsymbol{f}_{n, j}
\end{array}\right]
\end{gathered}
$$


Note that $\boldsymbol{W}_{-1, j}=\boldsymbol{W}_{0, j}=[0, \ldots, 0]^{T}$ because no real forces are applied to links $L_{-1}$ and $L_{0}$ (i.e. $\boldsymbol{f}_{-1, j}=\boldsymbol{f}_{0, j}=\mathbf{0}$ ). It is also worth noting that the dimension of $\mathcal{W}$ is $n+2$, and a generalized wrench $\boldsymbol{W}_{i, j}$ has therefore $m=n+2$ components, with $2+i$ non null components. In summary, the dimension of the generalized wrench is equal to the number of $d o f$ of the articulated object, i.e. $n+2$. The number of non null components is $i+2$, but in any case the maximum number of independent components is always three, originated from the two components $\mathrm{x}$ and $\mathrm{y}$ of $\boldsymbol{f}_{i, j}$, and from one parameter defining the contact point $\boldsymbol{p}_{i, j}$.

\subsection{Force-Closure Test}

As mentioned in Section 1, serial articulated objects with $n$ links have $m=n+2$ dof and can be inmobilized with $k=m+1=n+3$ frictionless contacts. Now, considering the set of $k$ contact points $G=\left\{\boldsymbol{p}_{i, j}, i=1, \ldots n, j=1, \ldots, k_{i}\right\}$ and a unitary force $\boldsymbol{f}_{i, j}$ applied at each $\boldsymbol{p}_{i, j}$, a set $W=\left\{\boldsymbol{W}_{i, j}, i=1, \ldots, n, j=1, \ldots, k_{i}\right\}$ is obtained. The necessary and sufficient condition for the existence of an FC grasp is that the origin of the generalized wrench space lies inside the convex hull of the contact wrenches $W$ [4][32]. The test used in this work to verify this condition is based on the following Lemma, derived from [13] for the case of a rigid object (linear programming techniques can also be used [33][34]).

Lemma. Let $G$ be a grasp with a set $W=\left\{\boldsymbol{W}_{i, j}, i=1, \ldots, n, j=1, \ldots, k_{i}\right\}$ of $k=n+3$ contact wrenches for an articulated object with $m=n+2$ dof. Let $H_{l}, l=1, \ldots, k$ be each of the hyperplanes defined in the wrench space by the set of points $W-\left\{\boldsymbol{W}_{i, j}\right\}$ with $i=1, \ldots, n, j=1, \ldots, k_{i}$, and let $P$ be the centroid of $W . P$ and the origin of the wrench space $O$ must lie on the same side of each hyperplane $H_{l}$ in order for grasp G to be FC. $\diamond$

Fig. 3 illustrates the Lemma for a hypothetical 2D wrench space. Given a grasp with a wrench set $W=\left\{\boldsymbol{W}_{1,1}, \boldsymbol{W}_{2,1}, \boldsymbol{W}_{3,1}\right\}$, hyperplanes $H_{l}$ are defined by the following sets of points:

$$
\begin{aligned}
& H_{1} \text { defined by } W-\left\{\boldsymbol{W}_{1,1}\right\}=\left\{\boldsymbol{W}_{2,1}, \boldsymbol{W}_{3,1}\right\} \\
& H_{2} \text { defined by } W-\left\{\boldsymbol{W}_{2,1}\right\}=\left\{\boldsymbol{W}_{1,1}, \boldsymbol{W}_{3,1}\right\} \\
& H_{3} \text { defined by } W-\left\{\boldsymbol{W}_{3,1}\right\}=\left\{\boldsymbol{W}_{1,1}, \boldsymbol{W}_{2,1}\right\}
\end{aligned}
$$

The lemma is fulfilled for the grasp in Fig. 3a because origin $O$ and $P$ are on the same side of each hyperplane $H_{l}$. The grasp in Fig. $3 \mathrm{~b}$ is not $\mathrm{FC}$ because origin $O$ and $P$ are on different sides of hyperplane $H_{3}$.

\section{Grasp analysis}

Using the results of the previous section, it is possible to analyze in a systematic way whether a given set of contact points on the articulated object allows an FC grasp.

As examples, consider an articulated object with two links, as shown in Fig. 4 with the grasping points $G=\left\{\boldsymbol{p}_{1,1}, \boldsymbol{p}_{1,2}, \boldsymbol{p}_{1,3}, \boldsymbol{p}_{2,1}, \boldsymbol{p}_{2,2}\right\}$. The grasp in Fig. 4a is not FC because the contact points on the second link generate moments of the same sign, and 

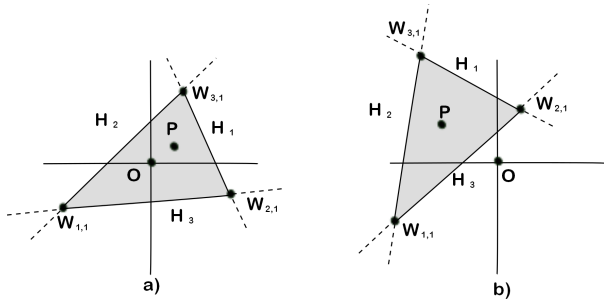

Figure 3: FC test in a hypothetical 2D space, a) FC grasp, b) non-FC grasp.

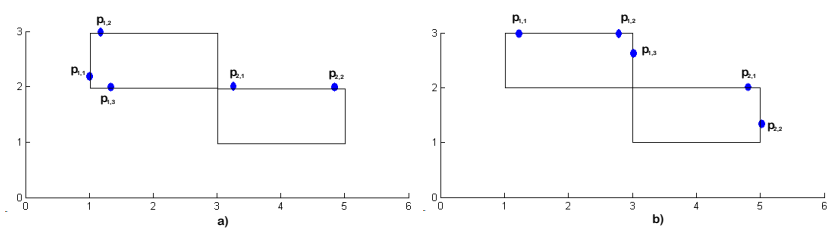

Figure 4: Illustration of two non-FC grasps.

external disturbances on the second link may therefore produce moments that cannot be counterbalanced.

The grasp in Fig. 4b is not FC because the contact points on both links cannot counterbalance any external force pushing the object down. The test in Lemma 1 is not fulfilled in either case.

If set $G$ allows an FC grasp, it means that the convex hull $(W)$ contains the origin of the generalized wrench space $\mathcal{W}$. Note that each generalized wrench $\boldsymbol{W}_{i, j}$ will have a number of independent components smaller than the dimension of $\mathcal{W}$ which depend on the link where the contact point is located. This imposes additional constraints on the distribution of forces applied on the object boundary, requiring them to be properly distributed on the object links (for instance, in the simple case of an object with two links and one joint it is straightforward that at least two contact points are necessary on each link).

Note that this approach is a generalization of those already used for grasp analysis of 2D and 3D rigid bodies using, respectively, 3-dimensional and 6-dimensional wrench spaces, even when here each generalized wrench $\boldsymbol{W}_{i, j}$ has a reduced number of independent components.

\section{Grasp Synthesis}

Following the developments above, the main idea of the algorithm described in this section is a generalization of that in [13]. This generalization considers that the wrench space may have any dimension other than 3 and 6 for 2D and 3D rigid objects, respectively. The algorithm generates a grasp $G^{1}$ by selecting $k$ random points from set $\Omega$, which describes the object boundary, then computes the corresponding set $W^{1}$ of generalized wrenches and verifies whether the points in $G^{1}$ allow an FC grasp. If it 

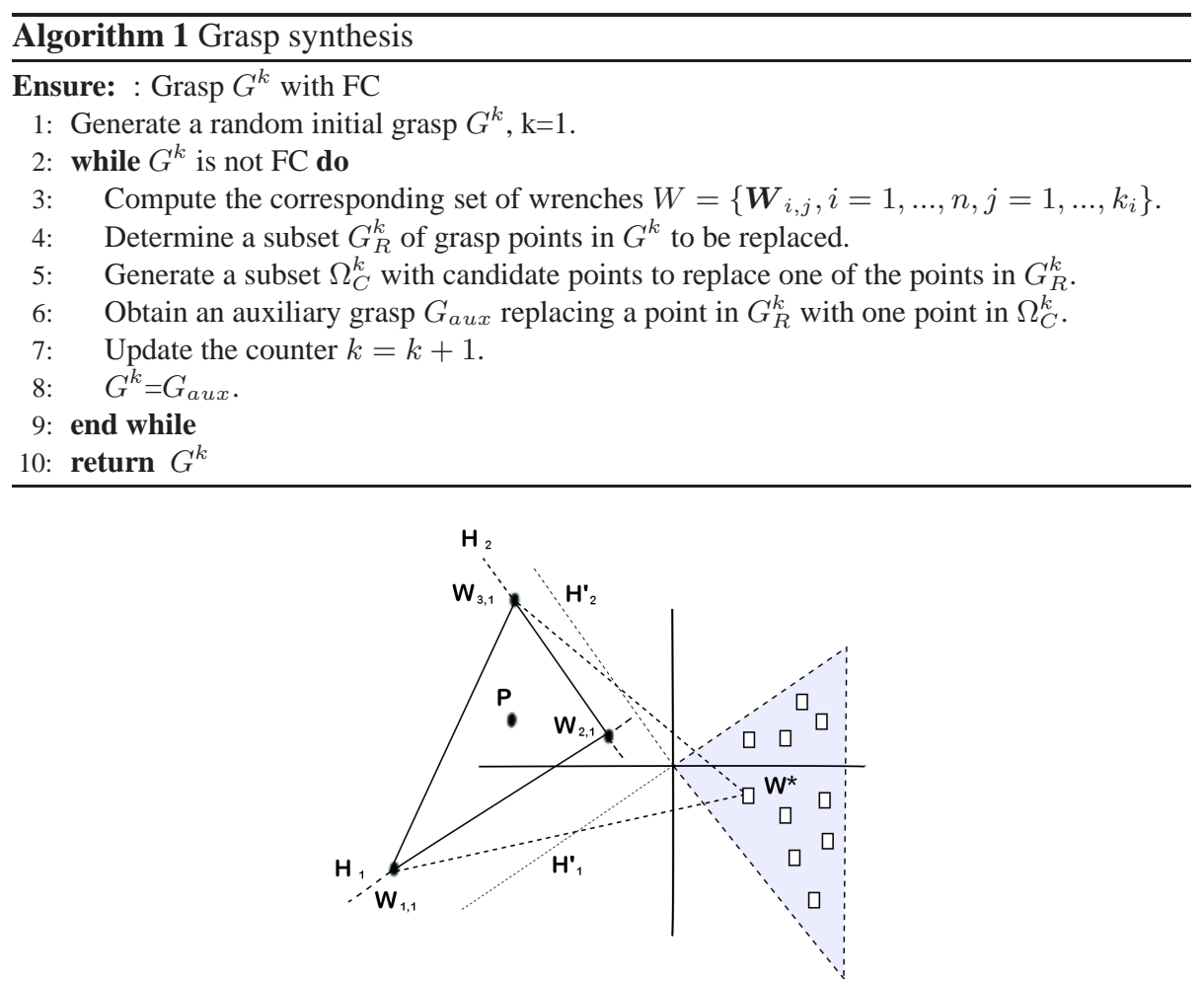

Figure 5: Search procedure to find one FC grasp in a hypothetical 2D wrench space.

does not, then a search for new contact points is done based on separating hyperplanes in the wrench space that define candidate points to replace one of the current points in $G^{1}$. This procedure is iteratively repeated until an FC grasp is found. The steps of the procedure, described in Algorithm 1, are explained below.

If grasp $G^{k}$ fails the FC test, the search procedure, steps (4) to (8) of the algorithm, iteratively tries to improve the grasp by changing one of the points in $G^{k}$.

In Step (4) subset $G_{R}^{k}$ is formed by all the wrenches in $W$ that simultaneously belong to all hyperplanes $H_{l}$ that cause the FC test failure, i.e. those hyperplanes not satisfying the condition in Lemma 1. If there is only one critical hyperplane, then $G_{R}^{k}$ includes all the points that define such hyperplane. Fig. 5 shows a hypothetical example in 2D (note that the real wrench space is $m$-dimensional): grasp $G$ producing wrenches $W=\left\{\boldsymbol{W}_{1,1}, \boldsymbol{W}_{2,1}, \boldsymbol{W}_{3,1}\right\}$ is not FC, with $H_{1}$ and $H_{2}$ being the hyperplanes that cause the FC test failure. Then, the set of possible points to be replaced is $G_{R}^{k}=$ $\left\{\boldsymbol{p}_{2,1}\right\}$, with $\boldsymbol{p}_{2,1}$ being the point that produces wrench $\boldsymbol{W}_{2,1}$, which belongs to $H_{1}$ and $H_{2}$.

In Step (5), subset $\Omega_{C}^{k}$ with candidate points to replace one of the points in $G_{R}^{k}$ is determined using hyperplanes $H_{l}^{\prime}$ passing through the origin and parallel to the critical hyperplanes $H_{l}$. Candidate points to be used for the replacement are those that simul- 
taneously lie on the opposite side of point $P$ with respect to hyperplanes $H_{l}^{\prime}$. In Fig. 5, the points that produce the wrenches in the gray area determined by hyperplanes $H_{1}^{\prime}$ and $H_{2}^{\prime}$ belong to $\Omega_{C}^{k}$.

In Step (6), one of the points in $G_{R}^{k}$ is replaced by a point producing a wrench $W^{*}$ randomly taken from $\Omega_{C}^{k}$. An auxiliary grasp $G_{\text {aux }}$ is obtained with the replacement producing the set of wrenches $\left\{\boldsymbol{W}_{1,1}, \boldsymbol{W}^{*}, \boldsymbol{W}_{3,1}\right\}$, as shown in Fig. 5. For the candidate grasp, centroid $P^{*}$ and distance $\left|\overline{P^{*} O}\right|$ are computed, using $P^{k}$ to represent the centroid of the set of wrenches $W$ in iteration $k$. If the relation $\left|\overline{P^{*} O}\right|<\left|\overline{P^{k} O}\right|$ is satisfied, then the auxiliary grasp is selected and the corresponding $W^{*}$ is used for the replacement. If all points in $G_{R}^{k}$ are verified and none of them reduces the distance $\left|\overline{P^{k} O}\right|$, the candidate $G^{*}$ that has the smallest distance $\left|\overline{P^{*} O}\right|$ is selected.

Finally, in steps (7) to (8), counter $k$ is updated and the selected point is included in the new grasp $G^{k}=G_{\text {aux }}$.

\section{Numerical Examples}

In this section, we present some numerical examples illustrating the grasp analysis and synthesis of articulated objects with two and three links. The proposed approach has been implemented using Matlab and C++ on a Intel Core2 Duo 2.0 GHz computer.

\subsection{Grasp Analysis Examples}

Example 1. Articulated object composed of two aligned ellipses $x_{1}=2 \cos (\theta)$ and $y_{1}=\sin (\theta)$ linked at the end of the major axis, with the first centered at $(2,1)$, as shown in Fig. 6.

Example 2. Articulated object composed of two unaligned ellipses $x_{1}=2 \cos (\theta)$ and $y_{1}=\sin (\theta)$ linked at the end of the major axis, with the first centered at $(2,1)$ and the second rotated an angle $\beta=22.5^{\circ}$, as shown in Fig. 7 .

Example 3. Articulated object composed of two aligned rectangles with side lengths 1 and 2 , linked by a vertex, with the first centered at $(1,1.5)$, as shown in Fig. 8.

Example 4. Articulated object composed of two unaligned rectangles with side lengths 1 and 2 , linked by a vertex, with the first centered at $(1,1.5)$ and the second rotated an angle $\beta=18^{\circ}$, as shown in Fig. 6.1.

Example 5. Articulated object composed of two symmetric polygons without parallel edges with side lengths 1.41 and 3.16 and an angle $18^{\circ}$ between the longest sides, linked by a vertex, as shown in Fig. 10.

Example 6. Articulated object composed of three ellipses $x_{1}=2 \cos (\theta)$ and $y_{1}=$ $\sin (\theta)$ linked at the end of the major axis, with the first centered at $(2,1)$, the second aligned and the third rotated an angle $\beta=22.5^{\circ}$, as shown in Fig. 11.

\subsection{Grasp Synthesis Examples}

Several examples illustrating the application of the grasp synthesis algorithm are given in Fig. 12 to 18; the articulated objects and their positions are straightforward from the 
figures and the description of the objects in the previous subsection. The initial and final wrenches and the number of required iterations are given in each example.

\section{Conclusions}

This paper proposes a procedure to model the generalized wrench space and a systematic procedure to analyze and synthesize FC grasps of 2D articulated objects considering frictionless contacts. The approach is illustrated for polygonal and non-polygonal articulated objects with two and three links. The dimension of the generalized wrenches for objects with $n$ links is always equal to the number of degrees of freedom of the articulated object $(n+2)$, but they have a maximum number of independent components equal to three., which are derived from the three independent components of the applied forces (the two components of the applied force and one component fixing the contact point). Using the proposed generalized wrench space, it is possible to analyze in a general and systematic way whether a given set of contact points on the links of an articulated object allows an FC grasp. The proposed generalized wrench space can also be used to find FC grasps of articulated objects using a generalization of procedures initially developed for rigid objects.

Future work includes the generalization of the approach for the case of frictional contacts, as well as for 3D articulated objects with frictionless and frictional contacts. Real experiments should be performed using, for instance, a two-handed robotic system. The search for optimal grasps that meet quality criteria and the generalization of the approach for closed-loop articulated objects are also future research topics.

\section{Acknowledgment}

This work was partially supported by the Spanish Government through projects DPI2010-15446, DPI2011-22471 and DPI2013-40882-P.

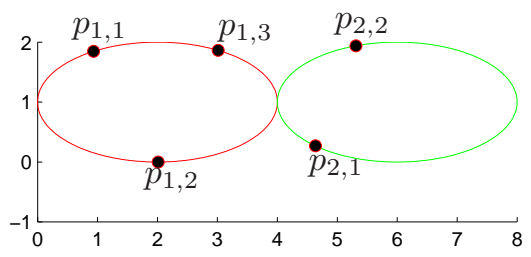

\begin{tabular}{|c|c|c|}
\hline Wrenches $\boldsymbol{W}_{i, j}$ & Points $\boldsymbol{p}_{i, j}$ & Normal $\hat{\boldsymbol{n}}_{i, j}$ \\
\hline $\boldsymbol{W}_{1,1}=\left[\begin{array}{llll}0.2774 & -0.9608-1.2010 & 0\end{array}\right]$ & $\boldsymbol{p}_{1,1}=\left[\begin{array}{ll}1 & 0.8660\end{array}\right]$ & $\boldsymbol{n}_{1,1}=[0.2774-0.9608]$ \\
\hline $\boldsymbol{W}_{1,2}=\left[\begin{array}{llll}0 & 1 & 2 & 0\end{array}\right]$ & $\boldsymbol{p}_{1,2}=\left[\begin{array}{ll}2 & -1\end{array}\right]$ & $\boldsymbol{n}_{1,2}=\left[\begin{array}{ll}0 & 1\end{array}\right]$ \\
\hline $\boldsymbol{W}_{1,3}=[-0.3088-0.9511-2.67930]$ & $\boldsymbol{p}_{1,3}=\left[\begin{array}{lll}3.0893 & 0.8387\end{array}\right]$ & $\boldsymbol{n}_{1,3}=[-0.3088-0.9511]$ \\
\hline $\boldsymbol{W}_{2,1}=\left[\begin{array}{llll}0.4855 & 0.8742 & 3.4970 & 0.7740\end{array}\right]$ & $\boldsymbol{p}_{2,1}=[0.5137-0.6691]$ & $\boldsymbol{n}_{2,1}=\left[\begin{array}{lll}0.4855 & 0.8742\end{array}\right]$ \\
\hline $\boldsymbol{W}_{2,2}=\left[\begin{array}{llll}0.1328 & -0.9911 & -3.9646 & -1.5975\end{array}\right]$ & $\boldsymbol{p}_{2,2}=\left[\begin{array}{lll}1.4824 & 0.9659\end{array}\right]$ & $\boldsymbol{n}_{2,2}=\left[\begin{array}{lll}0.1328 & -0.9911\end{array}\right]$ \\
\hline
\end{tabular}

Figure 6: Example 1 of grasp analysis. 


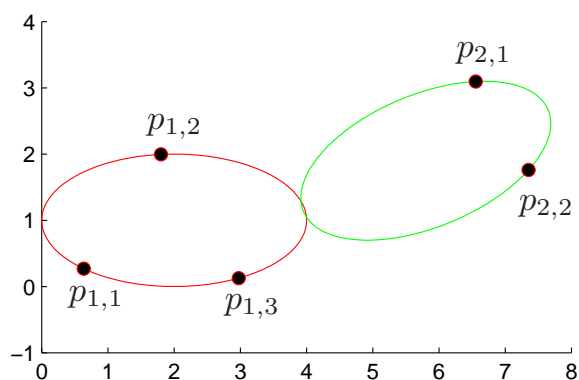

\begin{tabular}{|c|c|c|}
\hline Wrenches $\boldsymbol{W}_{i, j}$ & Points $\boldsymbol{p}_{i, j}$ & Normal $\hat{\boldsymbol{n}}_{i, j}$ \\
\hline $\boldsymbol{W}_{1,1}=\left[\begin{array}{llll}0.4472 & 0.8944 & 0.8402 & 0\end{array}\right]$ & $\boldsymbol{p}_{1,1}=\left[\begin{array}{ll}0.5858 & -0.7071\end{array}\right]$ & $\boldsymbol{n}_{1,1}=\left[\begin{array}{lll}0.4472 & 0.8944\end{array}\right]$ \\
\hline $\boldsymbol{W}_{1,2}=\left[\begin{array}{lll}0.0262 & -0.9997-1.92080\end{array}\right]$ & $\boldsymbol{p}_{1,2}=\left[\begin{array}{ll}1.8953 & 0.9986\end{array}\right]$ & $\boldsymbol{n}_{1,2}=\left[\begin{array}{lll}0.0262 & -0.9997\end{array}\right]$ \\
\hline $\boldsymbol{W}_{1,3}=\left[\begin{array}{llll}-0.2469 & 0.9690 & 2.5980 & 0\end{array}\right]$ & $\boldsymbol{p}_{1,3}=\left[\begin{array}{ll}2.9080 & -0.8910\end{array}\right]$ & $\boldsymbol{n}_{1,3}=\left[\begin{array}{lll}-0.2469 & 0.9690\end{array}\right]$ \\
\hline $\boldsymbol{W}_{2,1}=\left[\begin{array}{llll}0.0080 & -1 & -3.9999 & -2.7288\end{array}\right]$ & $\boldsymbol{p}_{2,1}=\left[\begin{array}{lll}2.7132 & 1.9651\end{array}\right]$ & $\boldsymbol{n}_{2,1}=[0.0080-1]$ \\
\hline $\boldsymbol{W}_{2,2}=\left[\begin{array}{llll}-0.7014 & 0.7127 & 2.8509 & 2.7288\end{array}\right]$ & $\boldsymbol{p}_{2,2}=\left[\begin{array}{lll}3.3080 & 0.5291\end{array}\right]$ & $\boldsymbol{n}_{2,2}=\left[\begin{array}{lll}-0.7014 & 0.7127\end{array}\right]$ \\
\hline
\end{tabular}

Figure 7: Example 2 of grasp analysis.

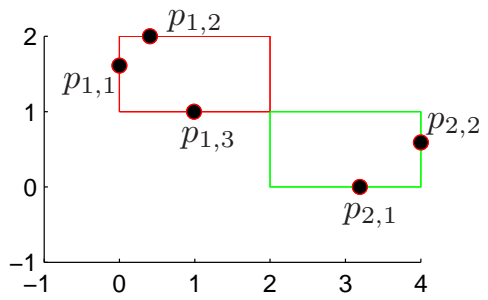

\begin{tabular}{|c|c|c|}
\hline Wrenches $\boldsymbol{W}_{i, j}$ & Points $\boldsymbol{p}_{i, j}$ & Normal $\hat{\boldsymbol{n}}_{i, j}$ \\
\hline $\boldsymbol{W}_{1,1}=\left[\begin{array}{lll}1 & 0 & -0.6316\end{array}\right]$ & $\boldsymbol{p}_{1,1}=\left[\begin{array}{ll}0 & 0.6316\end{array}\right]$ & $\boldsymbol{n}_{1,1}=\left[\begin{array}{ll}1 & 0\end{array}\right]$ \\
\hline $\boldsymbol{W}_{1,2}=\left[\begin{array}{llll}0 & -1 & -0.4103 & 0\end{array}\right]$ & $\boldsymbol{p}_{1,2}=[0.41031]$ & $\boldsymbol{n}_{1,2}=\left[\begin{array}{ll}0 & -1\end{array}\right]$ \\
\hline $\boldsymbol{W}_{1,3}=\left[\begin{array}{llll}0 & 1 & 0.9744 & 0\end{array}\right]$ & $\boldsymbol{p}_{1,3}=\left[\begin{array}{ll}0.9744 & 0\end{array}\right]$ & $\boldsymbol{n}_{1,3}=\left[\begin{array}{ll}0 & 1\end{array}\right]$ \\
\hline $\boldsymbol{W}_{2,1}=\left[\begin{array}{llll}0 & 1 & 2 & 1.1795\end{array}\right]$ & $\boldsymbol{p}_{2,1}=\left[\begin{array}{lll}1.1795 & -1\end{array}\right]$ & $\boldsymbol{n}_{2,1}=\left[\begin{array}{ll}0 & 1\end{array}\right]$ \\
\hline $\boldsymbol{W}_{2,2}=\left[\begin{array}{llll}-1 & 0 & -0.5 & -0.4211\end{array}\right]$ & $\boldsymbol{p}_{2,2}=\left[\begin{array}{ll}2 & -0.4211\end{array}\right]$ & $\boldsymbol{n}_{2,2}=\left[\begin{array}{ll}-1 & 0\end{array}\right]$ \\
\hline
\end{tabular}

Figure 8: Example 3 of grasp analysis.

\section{References}

[1] D. Prattichizzo, J. C. Trinkle, Handbook of robotics, Springer, 2008, Ch. Grasping, pp. 671-700.

[2] A. Bicchi, On the closure properties of robotic grasping, Int. J. Robotics Research 14 (4) (1995) 319-334.

[3] X. Markenscoff, C. H. Papadimitriou, The geometry of grasping, Int. J. Robotics Research 9 (1) (1990) 61-74.

[4] B. Mishra, J. Schwartz, M. Sharir, On the existence and synthesis of multifinger positive grips, Algorithmica 2 (4) (1987) 541-558. 


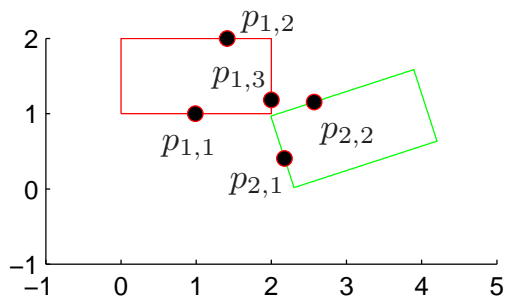

\begin{tabular}{|c|c|c|}
\hline Wrenches $\boldsymbol{W}_{i, j}$ & Points $\boldsymbol{p}_{i, j}$ & Normal $\hat{\boldsymbol{n}}_{i, j}$ \\
\hline $\boldsymbol{W}_{1,1}=\left[\begin{array}{llll}0 & 1 & 0.9744 & 0\end{array}\right]$ & $\boldsymbol{p}_{1,1}=\left[\begin{array}{lll}0.9744 & 0\end{array}\right]$ & $\boldsymbol{n}_{1,1}=\left[\begin{array}{ll}0 & 1\end{array}\right]$ \\
\hline 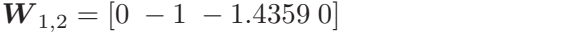 & $\boldsymbol{p}_{1,2}=\left[\begin{array}{lll}1.4359 & 1\end{array}\right]$ & $\boldsymbol{n}_{1,2}=\left[\begin{array}{ll}0 & -1\end{array}\right]$ \\
\hline $\boldsymbol{W}_{1,3}=\left[\begin{array}{llll}-1 & 0 & 0.1579 & 0\end{array}\right]$ & $\boldsymbol{p}_{1,3}=\left[\begin{array}{ll}2 & 0.1579\end{array}\right]$ & $\boldsymbol{n}_{1,3}=\left[\begin{array}{ll}-1 & 0\end{array}\right]$ \\
\hline $\boldsymbol{W}_{2,1}=\left[\begin{array}{llll}0.9511 & 0.3090 & 1.0935 & 0.5682\end{array}\right]$ & $\boldsymbol{p}_{2,1}=\left[\begin{array}{lll}0.1720 & -0.5415\end{array}\right]$ & $\boldsymbol{n}_{2,1}=\left[\begin{array}{ll}0.9511 & 0.3090\end{array}\right]$ \\
\hline $\boldsymbol{W}_{2,2}=\left[\begin{array}{ll}0.3090 & -0.9511-1.7477-0.6116\end{array}\right]$ & $\boldsymbol{p}_{2,2}=\left[\begin{array}{lll}0.5784 & 0.199\end{array}\right]$ & $\boldsymbol{n}_{2,2}=\left[\begin{array}{lll}0.3090 & -0.9511\end{array}\right]$ \\
\hline
\end{tabular}

Figure 9: Example 4 of grasp analysis.

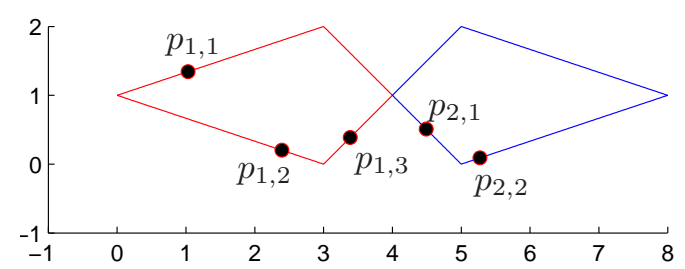

\begin{tabular}{|c|c|c|}
\hline Wrenches $\boldsymbol{W}_{i, j}$ & Points $\boldsymbol{p}_{i, j}$ & Normal $\hat{\boldsymbol{n}}_{i, j}$ \\
\hline $\boldsymbol{W}_{1,1}=[0.3162-0.9487-1.05410]$ & $\boldsymbol{p}_{1,1}=\left[\begin{array}{ll}1 & 0.3333\end{array}\right]$ & $\boldsymbol{n}_{1,1}=\left[\begin{array}{ll}0.3162-0.9487\end{array}\right]$ \\
\hline $\boldsymbol{W}_{1,2}=\left[\begin{array}{llll}0.3162 & 0.9487 & 2.5136 & 0\end{array}\right]$ & $\boldsymbol{p}_{1,2}=\left[\begin{array}{lll}2.3846 & -0.7949\end{array}\right]$ & $\boldsymbol{n}_{1,2}=\left[\begin{array}{ll}0.3162 & 0.9487\end{array}\right]$ \\
\hline 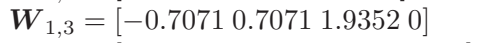 & $\boldsymbol{p}_{1,3}=[3.3684-0.6316]$ & $\boldsymbol{n}_{1,3}=\left[\begin{array}{lll}-0.7071 & 0.7071\end{array}\right]$ \\
\hline $\boldsymbol{W}_{2,1}=\left[\begin{array}{llll}0.7071 & 0.7071 & 2.8284 & 0.6699\end{array}\right]$ & $\boldsymbol{p}_{2,1}=[0.4737-0.4737]$ & $\boldsymbol{n}_{2,1}=\left[\begin{array}{ll}0.7071 & 0.7071\end{array}\right]$ \\
\hline $\boldsymbol{W}_{2,2}=\left[\begin{array}{llll}-0.3162 & 0.9487 & 3.7947 & 0.8757\end{array}\right]$ & $\boldsymbol{p}_{2,2}=\left[\begin{array}{lll}1.2308 & -0.9231\end{array}\right]$ & $\boldsymbol{n}_{2,2}=\left[\begin{array}{lll}-0.3162 & 0.9487\end{array}\right]$ \\
\hline
\end{tabular}

Figure 10: Example 5 of grasp analysis.

[5] Y. C. Park, G. P. Starr, Grasp synthesis of polygonal objects, in: Proc. IEEE Int. Conf. on Robotics and Automation, ICRA, Vol. 3, 1990, pp. 1574-1580.

[6] J. Ponce, D. Stam, B. Faverjon, On computing three-finger force-closure grasps of polygonal objects, IEEE Trans. on Robotics and Automation 11 (6) (1995) 868-881.

[7] J. Cornellà, R. Suárez, On 2d 4-finger frictionless optimal grasps, in: Proc. IEEE/RSJ Int. Conf. on Intelligent Robots and Systems, IROS, Vol. 3, 2003, pp. 3680 - 3685 .

[8] J. Cornellà, R. Suárez, Efficient determination of four-point form-closure optimal constraints of polygonal objects, in: IEEE Trans. on Automation Science and Engineering, 2009, pp. 121-130. 


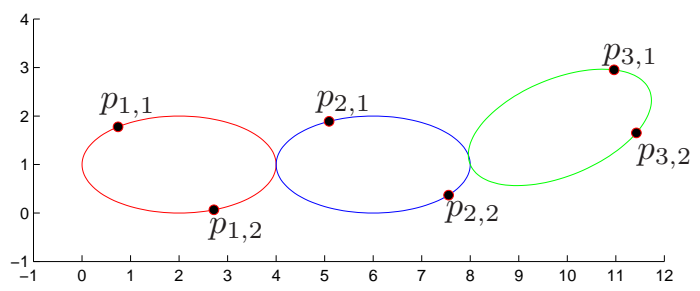

\begin{tabular}{|c|c|c|}
\hline Wrenches $\boldsymbol{W}_{i, j}$ & $\overline{\text { Points } \boldsymbol{p}_{i, j}}$ & $\overline{\text { Normal } \hat{\boldsymbol{n}}_{i, j}}$ \\
\hline $\boldsymbol{W}_{1,1}=\left[\begin{array}{llll}0.3753-0.9269-0.9788 & 0 & 0\end{array}\right]$ & $\boldsymbol{p}_{1,1}=[0.74140 .7771]$ & $\boldsymbol{n}_{1,1}=[0.3753-0.9269]$ \\
\hline $\boldsymbol{W}_{1,2}=\left[\begin{array}{lllll}-0.1885 & 0.9821 & 2.4921 & 0 & 0\end{array}\right]$ & $\boldsymbol{p}_{1,2}=[2.7167-0.9336]$ & $\boldsymbol{n}_{1,2}=\left[\begin{array}{lll}-0.1885 & 0.9821\end{array}\right]$ \\
\hline $\boldsymbol{W}_{2,1}=\left[\begin{array}{ll}0.2469-0.9690-3.8762-1.2782 & 0\end{array}\right]$ & $\boldsymbol{p}_{2,1}=\left[\begin{array}{ll}1.0920 & 0.8910\end{array}\right]$ & $\boldsymbol{n}_{2,1}=[0.2469-0.9690]$ \\
\hline $\boldsymbol{W}_{2,2}=\left[\begin{array}{lllll}-0.5254 & 0.8509 & 3.4035 & 2.6936 & 0\end{array}\right]$ & $\boldsymbol{p}_{2,2}=[3.5543-0.6293]$ & $\boldsymbol{n}_{2,2}=\left[\begin{array}{lll}-0.5254 & 0.8509\end{array}\right]$ \\
\hline 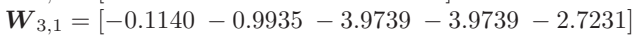 & $\boldsymbol{p}_{3,1}=\left[\begin{array}{ll}2.9649 & 1.9524\end{array}\right]$ & $\boldsymbol{n}_{3,1}=\left[\begin{array}{lll}-0.1140 & -0.9935\end{array}\right]$ \\
\hline $\boldsymbol{W}_{3,2}=\left[\begin{array}{lllll}-0.7555 & 0.6552 & 2.6208 & 2.6208 & 2.7376\end{array}\right]$ & $\boldsymbol{p}_{3,2}=\left[\begin{array}{lll}3.4250 & 0.6533\end{array}\right]$ & $\boldsymbol{n}_{3,2}=\left[\begin{array}{lll}-0.7555 & 0.6552\end{array}\right]$ \\
\hline
\end{tabular}

Figure 11: Example 6 of grasp analysis.
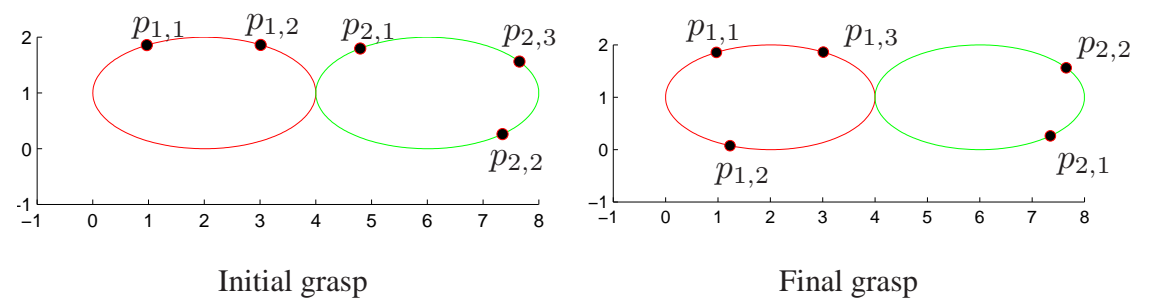

Final grasp

\begin{tabular}{|c|c|}
\hline Initial Wrenches & Final Wrenches \\
\hline 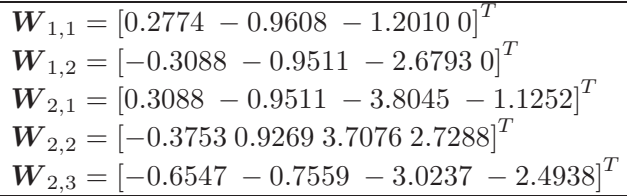 & 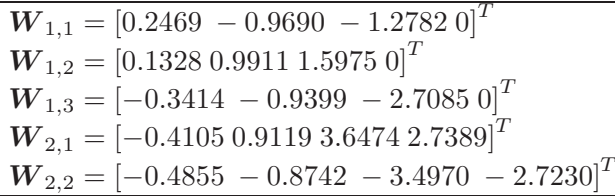 \\
\hline
\end{tabular}

Figure 12: Example 1 of grasp synthesis, articulated object with two ellipses, the FC grasp was achieved after 11 iterations.

[9] J. Cornellà, R. Suárez, On computing form-closure grasps/fixtures for nonpolygonal objects, in: Proc. IEEE Int. Symp. on Assembly and Task Planning, 2005, pp. 138-143.

[10] N. Niparnan, A. Sudsang, Computing all force-flosure grasps of 2d objects from contact point set, in: Proc. IEEE/RSJ Int. Conf. on Intelligent Robots and Systems, IROS, 2006, pp. 1599-1604.

[11] R. Prado, R. Suárez, Heuristic grasp planning with three frictional contacts on two or three faces of a polyhedron, in: Proc. IEEE Int. Symp. on Assembly and Task Planning, 2005, pp. 112-118. 

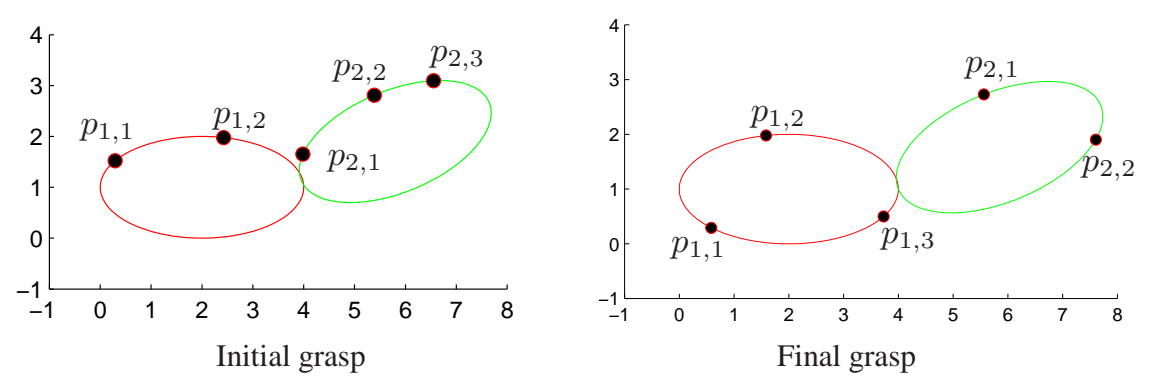

\begin{tabular}{|c|c|}
\hline Initial Wrenches & Final Wrenches \\
\hline $\boldsymbol{W}_{1,1}=\left[\begin{array}{ll}0.6101-0.7924-0.58790\end{array}\right]^{T}$ & $\boldsymbol{W}_{1,1}=\left[\begin{array}{lllll}0.4472 & 0.8944 & 0.8402 & 0\end{array}\right]^{T}$ \\
\hline $\boldsymbol{W}_{1,2}=\left[\begin{array}{lll}-0.1328 & -0.9911-2.36710\end{array}\right]^{T}$ & $\boldsymbol{W}_{1,2}=\left[\begin{array}{llll}-0.8668 & 0.4986 & 1.9943 & 2.5815\end{array}\right]^{T}$ \\
\hline $\boldsymbol{W}_{2,1}=\left[\begin{array}{ll}0.8668 & -0.4986-1.9943-0.5880\end{array}\right]^{T}$ & $\boldsymbol{W}_{1,3}=\left[\begin{array}{llll}-0.6547 & 0.7559 & 2.4938 & 0\end{array}\right]^{T}$ \\
\hline $\boldsymbol{W}_{2,2}=\left[\begin{array}{lll}0.3584 & -0.9336-3.7343-2.0778\end{array}\right]^{T}$ & $\boldsymbol{W}_{2,1}=\left[\begin{array}{lll}0.3584 & -0.9336-3.7343-2.0778\end{array}\right]^{T}$ \\
\hline $\boldsymbol{W}_{2,3}=[0.0080-1.0000-3.9999-2.7288]^{T}$ & $\boldsymbol{W}_{2,2}=[0.1057-0.9944-1.67870]^{T}$ \\
\hline
\end{tabular}

Figure 13: Example 2 of grasp synthesis, articulated object with two unaligned ellipses (the second rotated an angle $\beta=22.5^{\circ}$ ), the FC grasp was achieved after nine iterations.
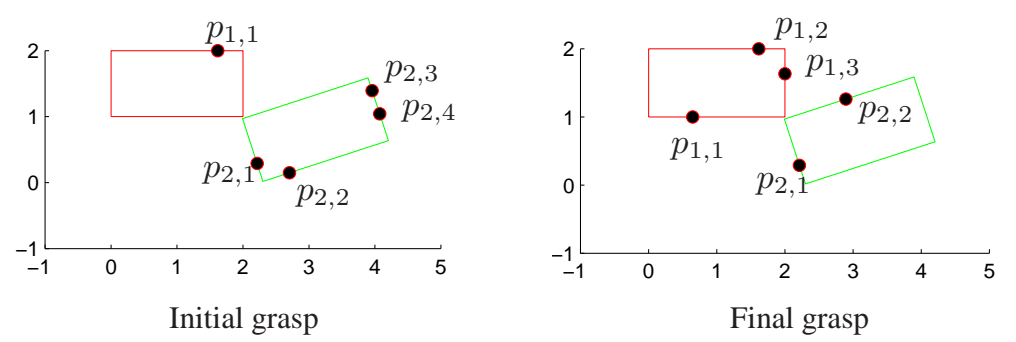

\begin{tabular}{|c|c|}
\hline Initial Wrenches & Final Wrenches \\
\hline $\boldsymbol{W}_{1,1}=\left[\begin{array}{lll}0 & -1.0000-1.6410 & 0\end{array}\right]^{T}$ & $\boldsymbol{W}_{1,1}=\left[\begin{array}{lllll}0 & 1.0000 & 0.6154 & 0\end{array}\right]^{T}$ \\
\hline $\boldsymbol{W}_{2,1}=\left[\begin{array}{llll}0.9511 & 0.3090 & 1.0935 & 0.7261\end{array}\right]^{T}$ & $\boldsymbol{W}_{1,2}=\left[\begin{array}{lll}0 & -1.0000-1.6410 & 0\end{array}\right]^{T}$ \\
\hline $\boldsymbol{W}_{2,2}=\left[\begin{array}{llll}-0.3091 & 0.9510 & 1.7476 & 0.4065\end{array}\right]^{T}$ & $\boldsymbol{W}_{1,3}=\left[\begin{array}{llll}-1.0000 & 0 & 0.6316 & 0\end{array}\right]^{T}$ \\
\hline 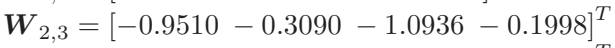 & $\boldsymbol{W}_{2,1}=\left[\begin{array}{llll}0.9511 & 0.3090 & 1.0935 & 0.7261\end{array}\right]^{T}$ \\
\hline $\boldsymbol{W}_{2,4}=[-0.9510-0.3090-1.0936-0.5682]^{T}$ & $\boldsymbol{W}_{2,2}=[0.3090-0.9511-1.7477-0.9706]^{T}$ \\
\hline
\end{tabular}

Figure 14: Example 3 of grasp synthesis, articulated object with two unaligned rectangles (the second rotated an angle $\beta=18^{\circ}$ ), the FC grasp was achieved after 10 iterations.

[12] R. Prado, R. Suárez, Synthesis of grasps with four contact points including at least three force-closure grasps of three contact points, in: Proc. IEEE/RSJ Int. Conf. on Intelligent Robots and Systems, IROS, 2008, pp. 1771-1776.

[13] M. Roa, R. Suárez, Finding locally optimum force-closure grasps, J. Robotics and Computer-Integrated Manufacturing 25 (3) (2009) 536-544.

[14] G. Dini, F. Failli, Planning grasps for industrial robotized applications using neu- 


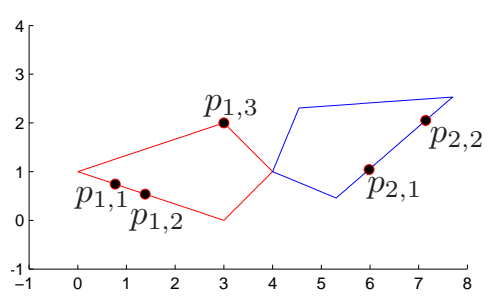

Initial grasp

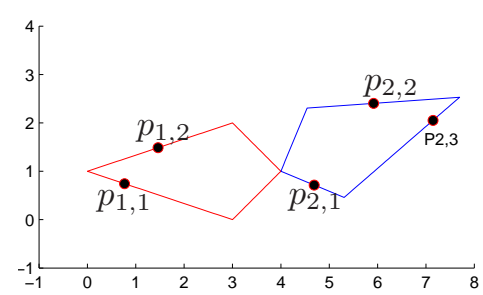

Final grasp

\begin{tabular}{|c|c|}
\hline Initial Wrenches & Final Wrenches \\
\hline $\boldsymbol{W}_{1,1}=\left[\begin{array}{llllll}0.3162 & 0.9487 & 0.8108 & 0\end{array}\right]^{T}$ & $\boldsymbol{W}_{1,1}=\left[\begin{array}{llllll}0.3162 & 0.9487 & 0.8108 & 0 .\end{array}\right]^{T}$ \\
\hline $\boldsymbol{W}_{1,2}=\left[\begin{array}{llll}0.3162 & 0.9487 & 1.4595 & 0\end{array}\right]^{T}$ & $\boldsymbol{W}_{1,2}=[0.3162-0.9487-1.54060]^{T}$ \\
\hline $\boldsymbol{W}_{1,3}=[0.3162-0.9487-3.16230]^{T}$ & $\boldsymbol{W}_{1,3}=\left[\begin{array}{lll}0.4471-0.8945-3.5780-2.3419\end{array}\right]^{T}$ \\
\hline $\boldsymbol{W}_{2,1}=\left[\begin{array}{llll}-0.8945 & 0.4472 & 1.7886 & 0.9242\end{array}\right]^{T}$ & $\boldsymbol{W}_{2,1}=\left[\begin{array}{llll}0.3825 & 0.9239 & 3.6958 & 0.744\end{array}\right]^{T}$ \\
\hline $\boldsymbol{W}_{2,2}=\left[\begin{array}{lllll}-0.8945 & 0.4472 & 1.7886 & 2.3475\end{array}\right]^{T}$ & $\boldsymbol{W}_{2,2}=\left[\begin{array}{llll}-0.8945 & 0.4472 & 1.7886 & 2.3475\end{array}\right]^{T}$ \\
\hline
\end{tabular}

Figure 15: Example 4 of grasp synthesis, articulated object with two unaligned polygons without parallel edges (the second rotated an angle $\beta=22^{\circ}$ ), the FC grasp was achieved after five iterations.

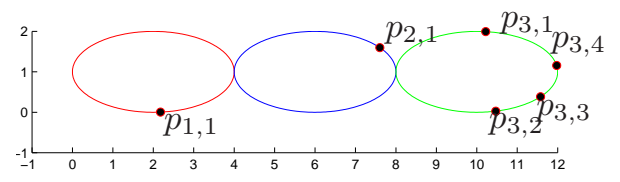

Initial grasp

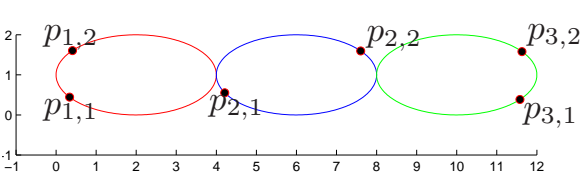

Final grasp

\begin{tabular}{|c|c|}
\hline Initial Wrenches & Final Wrenches \\
\hline $\boldsymbol{W}_{1,1}=\left[\begin{array}{llllll}-0.0262 & 0.9997 & 2.0778 & 0 & 0\end{array}\right]^{T}$ & $\boldsymbol{W}_{1,1}=\left[\begin{array}{llllll}0.6101 & 0.7924 & 0.5879 & 0 & 0\end{array}\right]^{T}$ \\
\hline $\boldsymbol{W}_{2,1}=[-0.6101-0.7924-3.1694-2.58150]^{T}$ & $\boldsymbol{W}_{1,2}=\left[\begin{array}{lllll}0.5254 & -0.8509 & -0.7099 & 0 & 0\end{array}\right]^{T}$ \\
\hline $\boldsymbol{W}_{3,1}=\left[\begin{array}{ll}-0.1328-0.9911-3.9646-3.9646-2.3671\end{array}\right]^{T}$ & $\boldsymbol{W}_{2,1}=\left[\begin{array}{lllll}0.7932 & 0.6090 & 2.4358 & 0.3652 & 0\end{array}\right]^{T}$ \\
\hline $\boldsymbol{W}_{3,2}=\left[\begin{array}{lllll}-0.0525 & 0.9986 & 3.9945 & 3.9945 & 2.1538\end{array}\right]^{T}$ & $\boldsymbol{W}_{2,2}=[-0.6101-0.7924-3.1694-2.58150]^{T}$ \\
\hline $\boldsymbol{W}_{3,3}=\left[\begin{array}{lllll}-0.4472 & 0.8944 & 3.5777 & 3.5777 & 2.7375\end{array}\right]^{T}$ & $\boldsymbol{W}_{3,1}=\left[\begin{array}{lllll}-0.4472 & 0.8944 & 3.5777 & 3.5777 & 2.7375\end{array}\right]^{T}$ \\
\hline $\boldsymbol{W}_{3,4}=\left[\begin{array}{lllll}-1.0000 & 0 & 0 ; & 0 & 0\end{array}\right]^{T}$ & $\boldsymbol{W}_{3,2}=\left[\begin{array}{llll}-0.7004 & -0.7137-2.8550-2.8550-2.3814\end{array}\right]^{T}$ \\
\hline
\end{tabular}

Figure 16: Example 5 of grasp synthesis, articulated object with three ellipses, the FC grasp was achieved after 55 iterations.

ral networks, J. Robotics and Computer-Integrated Manufacturing 16 (6) (2000) 451-463.

[15] N. Daoud, J. P. Gazeau, S. M. Zeghloul, M. Arsicault, A fast grasp synthesis method for online manipulation, J. Robotics and Autonomous Systems 59 (6) (2011) 421-427.

[16] S. El-Khoury, A. Sahbani, A new strategy combining empirical and analytical approaches for grasping unknown 3d objects, J. Robotics and Autonomous Systems 58 (5) (2010) 497507.

[17] K. Gopalakrishnan, K. Goldberg, D-space and deform closure grasps of deformable parts, Int. J. Robotics Research 24 (11) (2005) 899-910. 


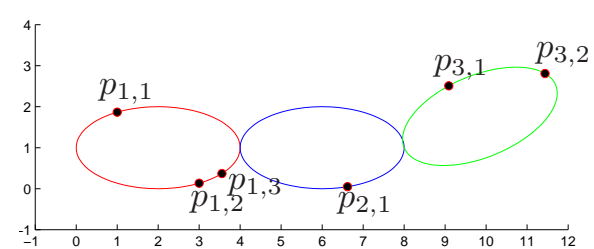

Initial grasp

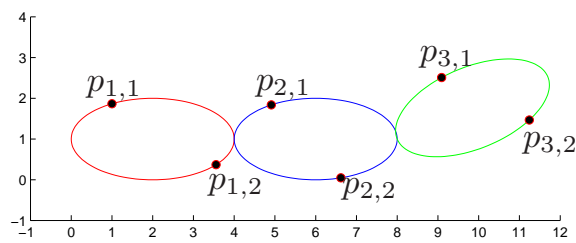

Final grasp

\begin{tabular}{|c|c|}
\hline Initial Wrenches & Final Wrenches \\
\hline $\boldsymbol{W}_{1,1}=\left[\begin{array}{lllll}0.2774-0.9608 & -1.2010 & 0 & 0\end{array}\right]^{T}$ & $\boldsymbol{W}_{1,1}=\left[\begin{array}{lllll}0.2774-0.9608-1.2010 & 0 & 0\end{array}\right]^{T}$ \\
\hline $\boldsymbol{W}_{1,2}=\left[\begin{array}{lllll}-0.2774 & 0.9608 & 2.6421 & 0 & 0\end{array}\right]^{T}$ & $\boldsymbol{W}_{1,2}=\left[\begin{array}{lllll}-0.5254 & 0.8509 & 2.6936 & 0 & 0\end{array}\right]^{T}$ \\
\hline $\boldsymbol{W}_{1,3}=\left[\begin{array}{llllll}-0.5254 & 0.8509 & 2.6936 & 0 & 0\end{array}\right]^{T}$ & $\boldsymbol{W}_{2,1}=[0.3088-0.9511-3.8045-1.12520]^{T}$ \\
\hline $\boldsymbol{W}_{2,1}=\left[\begin{array}{lllll}-0.1604 & 0.9871 & 3.9482 & 2.4316 & 0\end{array}\right]^{T}$ & $\boldsymbol{W}_{2,2}=\left[\begin{array}{lllll}-0.1604 & 0.9871 & 3.9482 & 2.4316 & 0\end{array}\right]^{T}$ \\
\hline $\boldsymbol{W}_{3,1}=[0.4782-0.8783-3.5131-3.5131-1.6787]^{T}$ & $\boldsymbol{W}_{3,1}=[0.4782-0.8783-3.5131-3.5131-1.6787]^{T}$ \\
\hline $\boldsymbol{W}_{3,2}=\left[\begin{array}{llll}-0.4998 & -0.8662-3.4646-3.4646-2.0707\end{array}\right]^{T}$ & $\boldsymbol{W}_{3,2}=\left[\begin{array}{lllll}-0.6751 & 0.7377 & 2.9508 & 2.9508 & 2.7086\end{array}\right]^{T}$ \\
\hline
\end{tabular}

Figure 17: Example 6 of grasp synthesis, articulated object with three unaligned ellipses (the third rotated an angle $\beta=22^{\circ}$ ), the FC grasp was achieved after 67 iterations.

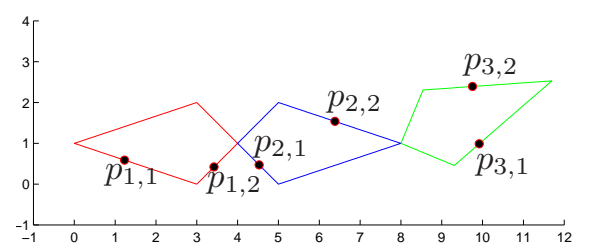

Initial grasp

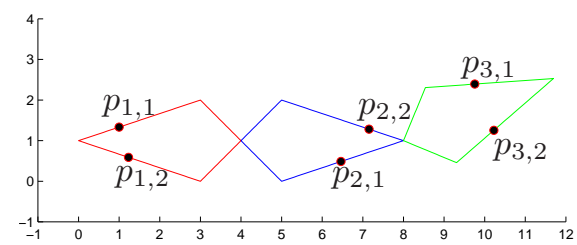

Final grasp

\begin{tabular}{|c|c|}
\hline Initial Wrenches & Final Wrenches \\
\hline $\boldsymbol{W}_{1,1}=\left[\begin{array}{llllll}0.3162 & 0.9487 & 1.2973 & 0 & 0\end{array}\right]^{T}$ & $\boldsymbol{W}_{1,1}=\left[\begin{array}{llllll}0.3162 & 0.9487 & 1.2973 & 0 & 0\end{array}\right]^{T}$ \\
\hline $\boldsymbol{W}_{1,2}=\left[\begin{array}{lllll}-0.7071 & 0.7071 & 2.0097 & 0 & 0\end{array}\right]^{T}$ & $\boldsymbol{W}_{1,2}=\left[\begin{array}{lllll}0.3162-0.9487 & -1.0541 & 0 & 0\end{array}\right]^{T}$ \\
\hline $\boldsymbol{W}_{2,1}=\left[\begin{array}{lllll}0.7071 & 0.7071 & 2.8284 & 0.7443 & 0\end{array}\right]^{T}$ & $\boldsymbol{W}_{2,1}=\left[\begin{array}{lllll}-0.3162 & 0.9487 & 3.7947 & 2.1731 & 0\end{array}\right]^{T}$ \\
\hline 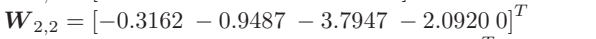 & $\boldsymbol{W}_{2,2}=[-0.3162-0.9487-3.7947-2.90280]^{T}$ \\
\hline $\boldsymbol{W}_{3,1}=\left[\begin{array}{lllll}-0.7071 & 0.7071 & 2.8284 & 2.8284 & 1.3500\end{array}\right]^{T}$ & $\boldsymbol{W}_{3,1}=\left[\begin{array}{ll}0.7071-0.7071-2.8284-2.8284-2.2254\end{array}\right]^{T}$ \\
\hline $\boldsymbol{W}_{3,2}=[0.7071-0.7071-2.8284-2.8284-2.2254]^{T}$ & $\boldsymbol{W}_{3,2}=\left[\begin{array}{lllll}-0.7071 & 0.7071 & 2.8284 & 2.8284 & 1.7544\end{array}\right]^{T}$ \\
\hline
\end{tabular}

Figure 18: Example 7 of grasp synthesis, articulated object with three unaligned polygons without parallel edges (the third rotated an angle $\beta=22^{\circ}$ ), the FC grasp was achieved after 37 iterations.

[18] Y.-B. Jia, F. Guo, H. Lin, Grasping deformable planar objects: Squeeze, stick/slip analysis, and energy-based optimalities, Int. J. Robotics Research 33 (6) (2014) 866-897.

[19] M. Bell, D. Balkcom, Grasping non-stretchable cloth polygons, Int. J. Robotics Research 29 (6) (2010) 775-784.

[20] A. Jain, G. Rodriguez, K. Kreutz-Delgado, Multi-arm grasp and manipulation of objects with internal degrees of freedom, in: Proc. IEEE Conf. on Decision and Control, Vol. 6, 1990, pp. 3110-3111. 


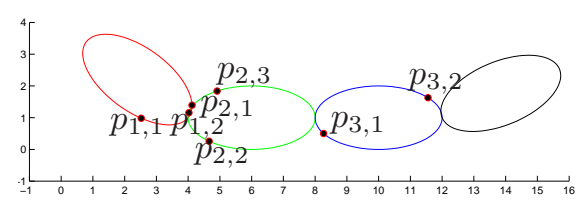

Initial grasp

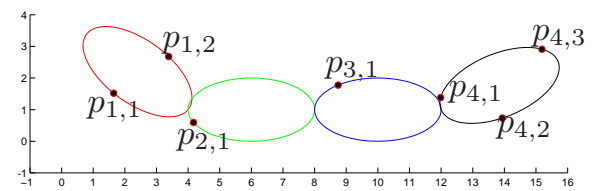

Final grasp

\begin{tabular}{|c|c|}
\hline Initial Wrenches & Final Wrenches \\
\hline $\boldsymbol{W}_{1,1}=\left[\begin{array}{llllllll}0.3979 & 0.9174 & 2.5477 & 0 & 0 & 0\end{array}\right]^{T}$ & $\boldsymbol{W}_{11}=\left[\begin{array}{lllllllll}0.6294 & 0.7771 & 1.8407 & 0 & 0 & 0\end{array}\right]^{T}$ \\
\hline $\boldsymbol{W}_{1,2}=\left[\begin{array}{llll}-0.9997-0.0264-2.0707 & 0 & 0 & 0\end{array}\right]^{T}$ & 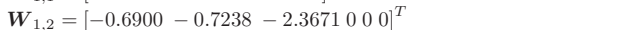 \\
\hline $\boldsymbol{W}_{2,1}=\left[\begin{array}{lllll}0.9533 & -0.3020 & 1.2641-0.1566 & 0 & 0\end{array}\right]^{T}$ & $\boldsymbol{W}_{2,1}=\left[\begin{array}{lllllll}0.7468 & 0.6650 & 3.9079 & 0.4187 & 0 & 0\end{array}\right]^{T}$ \\
\hline $\boldsymbol{W}_{2,2}=\left[\begin{array}{llllll}0.4105 & 0.9119 & 3.9160 & 0.9085 & 0 & 0\end{array}\right]^{T}$ & $\boldsymbol{W}_{3,1}=[0.3753-0.9269-2.1172-3.7076-0.97880]^{T}$ \\
\hline $\boldsymbol{W}_{2,3}=\left[\begin{array}{llll}0.3088 & -0.9511-2.3518-1.1252 & 0 & 0\end{array}\right]^{T}$ & $\boldsymbol{W}_{4,1}=[0.9659-0.25911 .4325-1.0363-1.0363-0.3652]^{T}$ \\
\hline 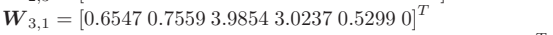 & $\boldsymbol{W}_{4,2}=\left[\begin{array}{llllll}-0.3086 & 0.9512 & 2.3528 & 3.8048 & 3.8048 & 1.7599\end{array}\right]^{T}$ \\
\hline $\boldsymbol{W}_{3,2}=[-0.5254-0.8509-3.9887-3.4035-2.69360]^{T}$ & $W_{4.3}=[-0.2604-0.9655-3.7367-3.8620-3.8620-2.5815]^{T}$ \\
\hline
\end{tabular}

Figure 19: Example 8 of grasp synthesis, articulated object with four unaligned polygons without parallel edges (the first and the fourth rotated an angle $\alpha=36^{\circ}$ and $\beta=22^{\circ}$, respectively), the $\mathrm{FC}$ grasp was achieved after 25 iterations.

[21] D. Katz, O. Brock, Manipulating articulated objects with interactive perception, in: Proc. IEEE Int. Conf. on Robotics and Automation, ICRA, 2008, pp. 272-277.

[22] X. Huang, I. Walker, S. Birchfield, Occlusion-aware reconstruction and manipulation of 3d articulated objects, in: Proc. IEEE Int. Conf. on Robotics and Automation, ICRA, 2012, pp. 1365-1371.

[23] J. S. Cheong, K. Goldberg, A. F. van der Stappen, Fixturing hinged polygons, in: Proc. IEEE Int. Conf. on Robotics and Automation, ICRA, Vol. 1, 2002, pp. 876-881.

[24] J. S. Cheong, A. F. van der Stappen, K. Goldberg, M. H. Overmars, E. Rimon, Immobilizing hinged polygons, International Journal of Computational Geometry \& Applications 17 (1) (2007) 45-69.

[25] E. Rimon, A. F. van der Stappen, Immobilizing 2-d serial chains in form-closure grasps, IEEE Trans. on Robotics 28 (1) (2012) 32-43.

[26] G. Carbone, A. González, A numerical simulation of the grasp operation by larm hand iv: A three finger robotic hand, J. Robotics and Computer-Integrated Manufacturing 27 (2) (2011) 450-459.

[27] M. Roa, K. Hertkorn, C. Borst, G. Hirzinger, Reachable independent contact regions for precision grasps, in: Proc. IEEE Int. Conf. on Robotics and Automation, ICRA, 2011, pp. 5337-5343.

[28] F. Gilart, R. Suárez, Determining force-closure grasps reachable by a given hand, in: IFAC Symposium on Robot Control, SYROCO, 2012, pp. 235-240.

[29] J. De Schutter, T. De Laet, Constraint-based task specification and estimation for sensor-based robot systems in the presence of geometric uncertainty, Int. J. Robotics Research 26 (5) (2007) 1-33. 
[30] B. Siciliano, L. Sciavicco, L. Villani, G. Oriolo, Robotics modelling, planning and control, Springer, 2009.

[31] J. J. Craig, Introduction to robotics: mechanics and control, Prentice Hall, 2004.

[32] R. M. Murray, Z. Li, S. S. Sastry, A mathematical introduction to robotic manipulation, CRC PressINC, 1994.

[33] Y. Liu, M. Lam, D. Ding, A complete and efficient algorithm for searching 3$\mathrm{d}$ form-closure grasps in the discrete domain, IEEE Trans. on Robotics 20 (5) (2004) 805-816.

[34] H. Asada, M. Kitagawa, Kinematic analysis and planning for form closure grasps by robotic hands, J. Robotics and Computer-Integrated Manufacturing 5 (4) (1989) 293-299. 